\title{
Pregnancy combined with severe pneumonia caused by Chlamydia psittaci infection - a case report
}

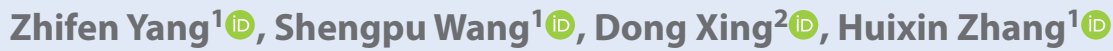 \\ ${ }^{1}$ Department of Obstetrics, the Fourth Hospital of Hebei Medical University, Shijiazhuang, Hebei, China \\ ${ }^{2}$ Department of Emergency, the Fourth Hospital of Hebei Medical University, Shijiazhuang, Hebei, China
}

\begin{abstract}
Objective: Chlamydia psittaci (Cps) feeds on gram-negative aerobic cells. Respiratory inhalation of $\mathrm{Cps}$ or close contact with infected animals can cause psittacosis in humans. Given its challengeable early diagnosis and rapid progression, misdiagnosis or improper antibacterial drugs may lead to multi-organ dysfunction with a high mortality.

Case report: A pregnant woman at $29^{+1}$ weeks of gestational age had a fever. CT images of the chest showed lamellar consolidations in the inferior lobe of the right lung, light inflammation in the middle lobe of the right lung. Negative results were obtained from multiple times of blood and sputum culture. Next generation sequencing (NGS) results identified that $C p$ s caused severe pneumonia, which was alleviated by specific antibiotic treatment.

Conclusion: Cps can cause severe pneumonia in the pregnant woman and be cured through precise antibiotic treatment. NGS is efficient in the rapid identification of pathogens and accurate diagnosis when bacteriological culture fails. Keywords: chlamydia psittaci; pregnancy combined with severe pneumonia; next generation sequencing; case report
\end{abstract}

\section{INTRODUCTION}

Chlamydia psittaci (Cps) is an obligate pathogenic organism living in gram-negative aerobic cells and surviving in vitro [1]. Misdiagnosis or improper antibacterial medication may lead to multi-organ dysfunction, with a relatively high mortality. Next Generation Sequencing (NGS) technique is efficient in rapid screening and identification of pathogens [2].

In this case report, we retrospectively analyzed a pregnant woman diagnosed with Cps-caused severe pneumonia by NGS.

\section{CASE PRESENTATION}

A 33-year-old pregnant woman at $29^{+1}$ weeks of gestational age (G3P2) presented to our department for 4-day fever. She felt chill, weakness, soreness after catchin, with developed symptoms of runny nose, nasal congestion and dry cough without symptoms of cough, expectoration, chest tightness and pain. The temperature continued to rise to $39.9^{\circ} \mathrm{C}$. So she was admitted to hospital. Emergency $\mathrm{CT}$ of the chest (Fig. $1 \mathrm{a}, \mathrm{b}$ ) showed lamellar consolidations in the inferior lobe of the right lung, slight inflammation in the middle lobe of the right lung, and little effusion in the right pleural cavity. Routine blood test showed blood cell
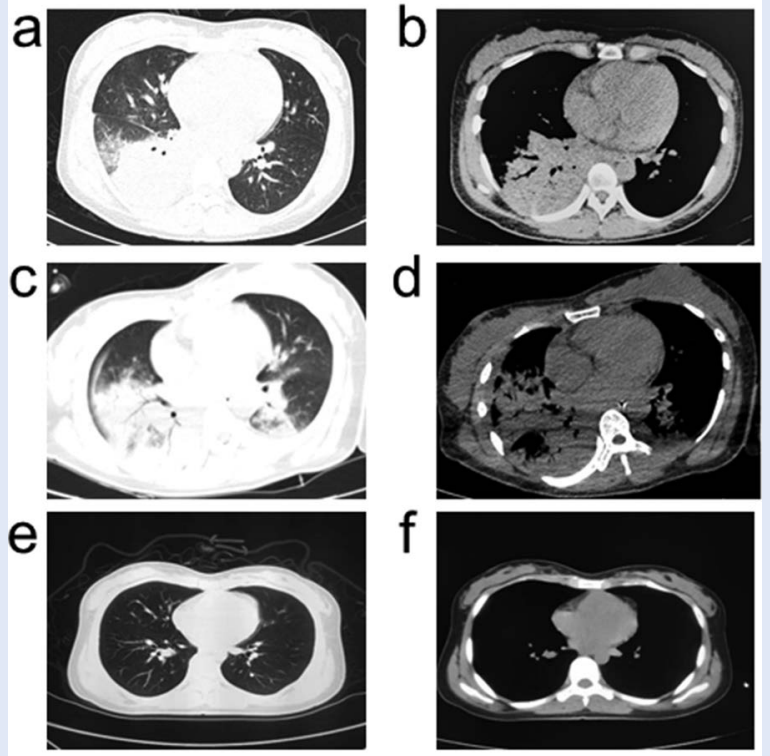

Figure 1. CT findings of lungs. a, b. Lamellar consolidations in the inferior lobe of the right lung, slight inflammation in the middle lobe of the right lung, and little effusion in the right pleural cavity on the $4^{\text {th }}$ day of onset. c, d. Multiple consolidations in both lungs, and little pericardial and pleural effusion. e, f. Improved pneumonia with clear lung fields and little inflammatory infiltration

\section{Corresponding author:}

Zhifen Yang, Department of Obstetrics, the Fourth Hospital of Hebei Medical University, Jiankang Road 12, Shijiazhuang, Hebei, 050011, China e-mail: cxsxfy@l 26.com, or Huixin Zhang, Department of Obstetrics, the Fourth Hospital of Hebei Medical University, Jiankang Road 12, Shijiazhuang, Hebei, 050011, China e-mail: zhx6519@163.com 
count (WBC), $7.79 \times 10^{9} / \mathrm{L}$; neutrophil granulocyte (NEU\%), 82.40\%; hemoglobin ( $\left.\mathrm{Hb}\right), 88 \mathrm{~g} / \mathrm{L}$; C-reactive protein (CRP), $116.54 \mathrm{mg} / \mathrm{L}$; procalcitonin (PCT), $0.07 \mu \mathrm{g} / \mathrm{L}$; blood urea nitrogen (BUN), $12.2 \mathrm{mmol} / \mathrm{L}$. Blood gas analysis showed pH, 7.455; $\mathrm{PO}_{2}, 55.1 \mathrm{mmHg} ; \mathrm{PCO}_{2}, 30.7 \mathrm{mmHg} ; \mathrm{BE},-2.7 \mathrm{mmol} / \mathrm{L} ; \mathrm{Lac}, 1.1 \mathrm{mmol} / \mathrm{L} ; \mathrm{FiO}_{2}, 0.21$. COVID-19 was excluded since the patient did not have a history of contacting or residing with COVID-19, and the nucleic acid test was negative. Cardiotocography (CTG) suggested an increased baseline rate of the fetal heart to 170 beats/min. No other abnormal findings were detected.

Oral Lianhuaqingwen capsules and intravenous cefamandole nafate were administered on the first day after admission. Negative results were obtained from urine and blood culture. The temperature fluctuated between $37.9^{\circ} \mathrm{C}-40.1^{\circ} \mathrm{C}$. The efficacy of anti-infective was unsatisfactory. Multidisciplinary consultations involving experts in pulmonology, ICU, pharmacy, obstetrics, pediatrics and imaging department suggested that the pneumonia had aggravated to a severity endangering the maternal and neonatal health, and recommended terminating the pregnancy. Meanwhile, blood and sputum samples were collected for screening pathogens by NGS. The patient was then surgically treated with lower segment cesarean section under general anesthesia. No abnormalities were found in the placenta, umbilical cord and amniotic fluid. After surgery, the patient's temperature started to fall and fluctuated between $36.5^{\circ} \mathrm{C}$ and $39.6^{\circ} \mathrm{C}$.

Chest CT after 2 days of surgery (Fig. 1 c, d) showed enlarged multiple consolidations in both lungs compared with last time, and little pericardial and pleural effusion. NGS of sputum samples identified $C p s$ as the pathogen. Through a detailed history review, the patient reminded of having two parrots at home. The parrots died a few days before she was ill. Therefore, a therapy involving meropenem, doxycycline and moxifloxacin was given to decimate Cps. Obvious alleviation of symptoms and reduction in temperature were observed 7 days later. Chest CT (Fig. 1 e, $\mathrm{f}$ ) showed an obvious absorption of pulmonary exudation. Finally, the patient was confirmed as pneumonia caused by Cps infection.

The newborn was 1,555 g in weight. The Apgar score was 7-10-10. The newborn was given non-invasive positive pressure ventilation, poractant alfa injection for fetal lung maturation and mezlocillin sodium for anti-infection. A negative result was obtained by NGS of sputum samples.

\section{DISCUSSION}

Pneumonia caused by Cps has no specific imaging features, and is always diagnosed depending on cell culture and serological tests. NGS rapidly and accurately identifies organisms by comparing sequencing data. Targeted anti-infective therapy based on NGS can significantly reduce the use of antibacterial agents and improve the prognosis [3]. Currently, Chlamydia infection cases have been reported with NGS-detected high nucleic acid sequences in secretion samples from the lung tissue and lower respiratory trac [4], NGS-detected Cps infection in blood and cerebrospinal fluid samples, however, is rarely reported. Latest studies have reported that pneumonia cases caused by Cps infection account for $1 \%$ of all community acquired pneumonia cases [5]. To our best knowledge, pneumonia caused by Cps infection in pregnant women has not been reported yet. In this case, Cps was not detected in the sputum sample of the newborn.

Collectively, identifying pathogen is the key event during the treatment. NGS can provide rapid and accurate detection of pathogens when negative results are obtained from multiple times of bacteriological culture.

\section{Ethics approval and consent to participate}

The patient and her husband signed the consent form (Chinese version) to all the treatments, examinations and procedures. We sincerely thank the family of the patient for their consent to publish this case report.

\section{Funding}

Hebei Health Commission Scientific Research Foundation, Grant/Award Number: 20190686.

\section{Conflicts of interest}

None.

\section{REFERENCES}

1. Corsaro D, Greub G. Pathogenic potential of novel Chlamydiae and diagnostic approaches to infections due to these obligate intracellular bacteria. Clin Microbiol Rev. 2006; 19(2): 283-297, doi: 10.1128/CMR.19.2.283-297.2006, indexed in Pubmed: 16614250.

2. Forbes JD, Knox NC, Peterson CL, et al. Highlighting Clinical Metagenomics for Enhanced Diagnostic Decision-making: A Step Towards Wider Implementation. Comput Struct Biotechnol J. 2018; 16: 108-120, doi: 10.1016/j.csbj.2018.02.006, indexed in Pubmed: 30026887.

3. Brown JR, Bharucha T, Breuer J. Encephalitis diagnosis using metagenomics: application of next generation sequencing for undiagnosed cases. J Infect. 2018; 76(3): 225-240, doi: 10.1016/j.jinf.2017.12.014, indexed in Pubmed: 29305150.

4. Gu L, Liu W, Ru M, et al. The application of metagenomic next-generation sequencing in diagnosing Chlamydia psittaci pneumonia: a report of five cases. BMC Pulm Med. 2020; 20(1): 65, doi: 10.1186/s12890-020-1098-x, indexed in Pubmed: 32178660.

5. Hogerwerf L, DE Gier B, Baan B, et al. Chlamydia psittaci (psittacosis) as a cause of community-acquired pneumonia: a systematic review and meta-analysis. Epidemiol Infect. 2017; 145(15): 3096-3105, doi: 10.1017/S0950268817002060, indexed in Pubmed: 28946931. 